\title{
Erratum to: Synthesis and Antimicrobial Activity of Novel $N^{3}, N^{5}$-Bis[1-(\{1-[2-((E)-benzylidene)hydrazinyl]- 1-oxo-3-phenylpropan-2-yl\}amino)-3-methyl-1-oxobutan- 2-yl]pyridine-3,5-dicarboxamides
}

\author{
A. E. Amr ${ }^{a, *}$, E. A. Elsayed ${ }^{b}$, and A. I. Al-Sayady ${ }^{b}$ \\ ${ }^{a}$ Pharmaceutical Chemistry Department, Drug Exploration and Development Chair (DEDC), College of Pharmacy, \\ King Saud University, Riyadh, 11451 Saudi Arabia \\ ${ }^{b}$ Zoology Department, Faculty of Science, King Saud University, Riyadh, 11451 Saudi Arabia \\ *e-mail:aamr@ksu.edu.sa
}

Received July 31, 2021; revised July 31, 2021; accepted July 31, 2021

DOI: $10.1134 / \mathrm{S} 1070363221070240$

The affilation of authors in PDF version should read as

a Pharmaceutical Chemistry Department, Drug Exploration and Development Chair (DEDC), College of Pharmacy, King Saud University, Riyadh, 11451 Saudi Arabia

${ }^{b}$ Zoology Department, Faculty of Science, King Saud University, Riyadh, 11451 Saudi Arabia

The original article can be found online at https://doi.org/10.1134/S1070363221060177 